\title{
Developing Experimental of Dimple Effect Applied on Flat Plate against Fluid Flow
}

\author{
Irfan Syarif Arief ${ }^{1}$, Rachmadi Wahyu Adjie ${ }^{2}$ \\ (Received: 28 July 2017 / Revised: 24 June 2018 / Accepted: 28 September 2019)
}

\begin{abstract}
These concaves turn out to have an effect on the movement of the golf ball so as to help the golf ball fly further. From the advantages of golf balls, whether the cavity can help to reduce the friction of the hull with the water surface. This study aims to determine the effect of dimple on flat plate with different types of flow (laminar, transition, and turbulent) and how it affects the coefficient of friction (Cf). In addition, with the difference in the ratio of the concavity (Rc) how it affects the coefficient of friction (Cf). From these influences later we can know how the optimal dimple design on the hull of the ship. The simulation method will be done with Numeca Fine Open. This software can simulate the complicated shape dimple well than any other software. The result of the analysis will be known to decrease the coefficient of friction (Cf) during laminar flow, transition, and turbulent. With a growing influence when turbulent flow. In addition, the greater the ratio of the concentration will decrease the coefficient of friction greater than the small-scale ratio.
\end{abstract}

Keywords - dimple, flat plate, computional fluid dynamic, coefficient of friction, ratio of concavity.

\section{INTRODUCTION}

$\mathrm{D}$ evelopment of fluid mechanics science from time to time more and more rapidly. Similarly, the application of fluid mechanics science, both in the industrial world, the maritime world and in daily life. One of the parts studied in the science of fluid mechanics is about the external flow that crosses a body. An example is the flow of fluid that crosses a solid ball.

Knowledge of the fluid flow characteristic that flows over a body will be expected to be used in the maritime field. It is expected that with this knowledge, the construction of ship building can be engineered in such a way that can be obtained an ideal ship building construction and minimal will be friction resistance. A fluid that flows over an object, there will be friction force in it. This frictional force will be very disadvantageous and as much as possible to minimize. One effort made to minimize the frictional force is to modify the shape of the object itself [1].

The underlying fact of this research is an article about golf ball analysis that has uneven surface alias concaveconcave. Apparently this has a special purpose, namely that the distance traveled can be further. The basin is useful for reducing the air resistance in order to give the ability of the golf ball to slide further than previous research on the analysis of basins on the plate that can reduce the frictional force.

Based on the above the authors try to apply on flat plate which later expected from this research can be useful for maritime industry, especially in the construction of ship building construction. Fluid flow divided into 2 (two)

Irfan Syarif Arief, Department of Marine Engineering, Institut Teknologi Sepuluh Nopember (ITS), Surabaya, 60111, Indonesia. irfansya@its.ac.id

Rachmadi Wahyu Adjie, Department of Marine Engineering, Institut Teknologi Sepuluh Nopember (ITS), Surabaya, 60111, Indonesia. wahyu.adjie12@mhs.ne.its.ac.id / adjie.raptor@gmail.com types. But in science, it can divided into 3 state (Laminar, Transition, and Turbulent).

Laminar flow is the fluid flow shown by the motion of the fluid particles parallel and the fine current lines. In the laminar stream, fluid particles appear to move along smooth and smooth trajectories, with one layer gliding smoothly on adjacent layers. The viscosity properties of liquids play an important role in the formation of laminar flow. The flow of the laminer is steady meaning the flow is fixed. "Fixed" indicates that across the water flow, the flow discharge is fixed or the flow rate does not change over time.

The flow of fluid in the pipe, beginning with the flow of laminer and then in the next phase of the flow turned into turbulent flow. The phase between the laminer becomes turbulent is called the transition stream. The laminer stream follows Newton's law of the viscosity that connects the shear stress with the rate of change in the angular shape. But at low viscosity and high velocity laminar flow is unstable and turned into turbulent flow. Conclusions about the characteristics of the laminar flow are: fluid moves along a straight line, low fluid velocity, high viscosity and regular fluid motion trajectories between one another [2].

Turbulent flow is relatively large flow velocities will produce non-laminar flow but complex, irregular motion paths of particles between each other. Thus, the characteristics of turbulent flow: no regularity in the fluid path, mixed flow, high fluid speed, large-scale flow length and low viscosity. Characteristics of turbulent flow are shown by the formation of vortices in the flow, which results in a continuous mixing of particles of liquid particles across the flow cross section [3].

To distinguish the flow of whether turbulent or laminar, there is an unity number called the Reynolds Number. According to Reynold's experimental results, if the 
Reynolds number is less than 2000 , flow is usually a laminar flow. If the Reynolds number is greater than 4000 , the flow is usually turbulent. Medium between 2000 and 4000 streams can be laminary or turbulent depending on other factors that affect [4][5].

Reynolds number can be calculated by the following equation:

$R e=\frac{4 v R}{\vartheta}$

where, $R e$ is the Reynolds Number, $v$ is Flow Velocity, $R$ is the Radius and $\theta$ is the Kinematic Viscosity.

\section{A. Boundary Layer}

Boundary layer is a concept for the inhibited flow, first introduced by Prandtl in 1904. The boundary layer can be found on a fluidized cross section, the velocity profile at the beginning of the cross section will be uniform, and then gradually throughout the cross section will undergo a change in velocity profile Because the friction forces have slowed the fluid near the cross-sectional wall. Fully developed full speed is reached. At the entrance area, the fluid near the center of the cross-section appears to be unaffected by friction. While the fluid on the wall has been affected by friction.

Moving region of the plate side at the viscosity observation site is called the boundary layer. The first development of the boundary layer is laminar, but at a critical distance from the initial side of the plate, depending on the flow field and fluid properties, the disturbance will be strengthened, and the transition process occurs until turbulent flow. The turbulent area can be described as a shake force that works so that the fluid part will move back and forth.

The critical Reynolds number for the flow transition from laminar to turbulent is theoretically taken $4 \times 10^{5}$, in practice this price depends on the condition of surface roughness and the degree of free flow turbulence. The normal range for the start of the transition region is between $4 \times 10^{5}$ to $10^{6}$.

In the presence of enormous disturbances in the flow, the transition may begin to occur in the Reynolds number as low as $10^{5}$, and for streams independent of the fructose, the transition region may occur at Reynolds $2 \times 10^{6}$ or more.

\section{B. Drag}

Resistant force is a fluid force component of a body in the direction of the fluid flow or the movement of the object. The resistance styles are divided into form drag and wave drag. With the approach that in the flow does not arise the discussion of the drag force is only a form of resistance only, here in after referred to as drag.

Dimensionless parameter is expressed as the drag coefficient, $\mathrm{C}_{\mathrm{D}}$ in the equation below:

$C_{D}=\frac{F_{D}}{\frac{1}{2} \rho U^{2} \frac{\pi}{4} d^{2}}$

where, $C_{D}$ is the Coefficient of Drag, $F_{D}$ is the drag force, $\rho$ is the density, $\pi$ is coefficient 3,14 and $d$ is the diameter.

There are two drag that occurs on the golf ball, first is skin friction drag (drag force due to air friction with the ball) and pressure drag (inhibition force due to vortex flow behind the ball). On the slippery balls, the flow from the front will split the ball around the ball, moving backward, but the flow is released before it reaches the rear end, and there are small repetitions behind the ball. The flow is laminar flow. In a golf ball that has dimple, the release of this flow can be delayed, meaning the point of discharge flow can be shifted more backward, even fewer vortex.

The flow on the ball with dimple is turbulent flow. Pressure drag on turbulent flow is smaller than laminary flow. So, by giving a dimple to the ball (adding roughness) does increase the skin friction drag, but the reduction / reduction of pressure drag is much greater, so the total drag is smaller.

\section{Lift}

Lifting force is the resultant component of the fluid force perpendicular to the fluid flow. The magnitude of the lifting force for lifting objects with lift plane is generally defined as:

$F_{L}=\frac{C_{L} \rho U^{2} \frac{\pi}{4} d^{2}}{2}$

The lifting areas are forms which are capable of producing lift power such as: kites, aerofoils, hydrofoils, propellers or fans. From the equation above then the equation coefficient of lift is:

$C_{L}=\frac{F_{L}}{\frac{1}{2} \rho U^{2} \frac{\pi}{4} d^{2}}$

where, $C_{L}$ is the coefficient of lift, $F_{L}$ is the lift force, $\rho$ is the density, $\pi$ is the coefficient 3,14 and $d$ is the diameter.

\section{Vortex}

At the time of separation, the flow will be separated from the wall and form a vortex which then the vortex can also be shedding carried by the flow. This shedding mechanism can occur alternately and periodically, depending on the Reynolds number regime. Vortex shedding is caused because two vortices on the opposite side of wake affect each other.

An explanation of the vortex shedding mechanism, where there are vortex A and B pairs where vortex A grows first and larger so as to attract votrex $\mathrm{B}$, since vorticity $\mathrm{A}$ and $\mathrm{B}$ are opposite marks, then at some point the vorticity of B cuts the supply of vorticity A and results in Vortex A shedding. After A shedding behind it is formed again vortex $C$, which is then because vortex $B$ is larger then attracted towards B and the same thing in vortex A previously happened to vortex B, so the next mechanism repeats.

Vortex shedding also produces a fluctuation in the forces acting on the object. The forces will fluctuate at the frequency of the shedding. The frequency characteristics of a geometry can be represented by a dimensionless number. Strouhal number, St, whose value also depends on the Reynold number. The strouhal number value is given by:

$S t=\frac{f D}{v}$

where, $S t$ is the Strouhal number, $f$ is the frequency $(\mathrm{Hz})$, $D$ is the vortex distance (m) and $v$ is the fluid v Coefficient of Friction elocity $(\mathrm{m} / \mathrm{s})$.

Shifting dots for flow through a ball or circular cylinder. The circular particles around the boundary at A move faster in the turbulent boundary layer than in the laminar 
boundary layer. Thus, those particles have more kinetic energy to overcome unfavorable pressure gradients (pressure rises) on the back of the ball or cylinder. Therefore, the particles in the turbulent boundary layer are able to move farther back before they stop and separate from the boundary. As a result, the wake there is smaller, so are the obstacles. The boundary can be turbulent on the Reynolds number lower than this price as long as the ball or cylinder is rough or when the level of turbulence or intensity.

In the 1920 s, the level or intensity of turbulence in the sub-sonic wind tunnel was measured by finding the critical value of the $\mathrm{ReD}$ when the resistance was suddenly reduced (or when the drag coefficient became 0.3 for example) for a smooth ball mounted on a measuring system Style in the wind tunnel. The higher the intensity of turbulence, the lower the critical Reynolds number. For that time, this tool has been amazing. In subsequent developments, the new velocity findings have enabled us to study the fluctuations and intensities of turbulence in more detail.

\section{E. Dimple}

Ball that used in golf sports has a special shape and configuration, namely the surface that has small dimpledimple. This form turned out to have an influence on the aerodynamics of the golf ball. The golf ball is made perforated to make the layer shift in the air slower because there is a small layer on the groove / hole ball on the surface so as to extend the distance of the air layer shift in the closest layer on the surface, so the air velocity on the surface rises so that the speed difference with the layer above smaller. It can also reduce the incidence of turbulence at the end of the ball.

Generally golf balls have 300-500 dimple with an average depth of about 0.01 inches. Lift (lift) style and drag on the golf ball is very sensitive to the depth dimple.

Then from previous research that discussed the effect of dimple on flat plate, got some new formula which can be used in design of dimple (dimple) on flat plate to reduce friction force that happened in flat plate. Then then the formulas can be seen in the discussion below.

Ratio of diameter dimple. This ratio is the ratio between the magnitude of the dimple diameter to be designed with the squares of the dimple forming rectangles. This will be useful later to determine how much between the dimple one with the other dimple. The formula is as follows:

$R_{D S}=\frac{b}{s}$

where, $R d s$ is the ratio diameter of dimple, $b$ is the diameter dimple and $s$ is the square length.

The ratio of the concavity is the ratio between the diameter dimple or hole to be designed against the thickness of the plate. This ratio will affect the depth of dimple depth from plate thickness. In this case to meet the first requirement, then the dimple diameter to be made is half the thick plate.

$R_{c}=\frac{b}{2 r}$

where, $R_{C}$ is the ratio of concavity, $b$ is the diameter of dimple and $r$ is the radius of dimple.
From the ratio of dimple diameter and the ratio of concavity, the best value of the experimental results and simulation of previous research. For Rage Cage Ratio $\left(\mathrm{R}_{\mathrm{DS}}\right)$ studied by Mohammad Aziz Almujahidin, 2012. The best ratio in the experiments on this flat plate is 0.8 adjusted to the existing device in the CNC Laboratory, Department of Shipping System Engineering FTK ITS.

Meanwhile, from the simulation results conducted by Nizar Puji Kurniawan, 2011. That the best ratio of dampness is 0.3 so that these two results become the benchmark of the initial design of the basin experiment (dimple) on flat plate before.

In the current study, this study focuses on the effect of a single dimple on a flat plate. With this study we will see whether dimple reduces the coefficient of friction $(\mathrm{Cf})$ compared to normal flat plate, or not. In addition, the influence of the type of flow (laminar, transition, and turbulent) does also affect the coefficient of friction (Cf) created on flat plate.

In addition to the type of flow and single dimple, in this study will be used 4 pieces of dimple model with a different ratio of kecekungan. These four models will then be compared with flat plates without dimple to see how big the effect of single dimple on this flat plate and to find the optimum dimple.

\section{METHOD}

\section{A. Literature Review}

At this stage a literature study was conducted on various references related to the research topic. This literature study is intended to find the right concepts and methods to solve problems that have been formulated in the previous stage and to realize the intended purpose. This literature study includes looking for references to related theories or previous research results on the application of dimple on a flat plate.

In the literature study conducted in this research, it should be noted that the reference in conducting this research is obtained from two previous studies conducted by Nizar Puji Kurniawan, 2011 and Mohammad Aziz Almujahidin, 2012 regarding the dimple on flat plate. While the focus of current research is to know how big the influence of the type of flow (laminar, transition, and turbulent) on $\mathrm{Cf}$ which will come out as the output of the simulation of this research. In addition, the difference in the ratio of kecekungan also does participate influential on the types of flow.

Aside from these two final tasks, the literature sources used are the results of the experiments dimple on the car as well as the papers relating to the dimple on the flat plate.

\section{B. Design Parameter}

In this study, the designs to be manufactured must meet certain requirements. It aims to limit in terms of design creation and provide focus so that the design results can approach the desired value in the end result.

The first time we do is to calculate the Froude number. Froude number is useful in measuring the resistance of an object moving in water. This Froude number has no dimensions so it is more appropriate to use in model scale that we will do on this plate model so as to facilitate the 
simulation process. Then the number of Froude number to be used is between 0.2 to 0.6 .

Froude number calculation we use to find the speed with the initial design length is $125 \mathrm{~mm}$ with a diameter of 6 mm dimple. After being calculated, the Reynold number obtained is above 4000 so that the length of the plate is reduced repeatedly to obtain an ideal length of $24 \mathrm{~mm}$ where $6 \mathrm{~mm}$ as the diameter of the dimple.

\section{Drawings}

In this study 2D computer modeling is done with Autocad software, then drawn in 3D using CAD 3D software and then simulated on the computer model with Numeca Fine Open software.

The $2 \mathrm{D}$ drawing process using CAD is used to determine the dimensions of the hole diameter, the depth of the hole, and the curvature that will be created in the 3D model later. And based on 2D's description of Autocad it's easier to fix dimensional errors if they happen to CAD 3D.

Furthermore, after we describe the dimple, we continue the depiction on CAD 3D. The first step we make a beam size 24 × 20 × 20 millimeters which later became the domain or as a place of calculation of flow through the surface dimple. After that we add dimple with the direction facing the beam because the simulated later is the inner surface of the beam with the addition of dimple and without dimple.

After this step, we save the box shape with this additional dimple into the parasolid format (.x_t). Why save needed in parasolid format. This is done to facilitate the description of the shape in Fine Open. By importing images in the parasolid format we will facilitate in the process of depiction and editing of the model at the Numeca Fine Open, Hexpress facility (used for modeling, domain, and meshing manually) so as to make it faster in the meshing process.

The next stage is to switch to Numeca Fine Open, where we will create domains and meshing on the model we have drawn in $3 \mathrm{D}$ previously in CAD 3D. At the time of opening the software Numeca Fine Open will be directed to create a new mesh or open mesh that has been made. In this first step we have to create a new mesh via Hexpress on Numeca. This step will run automatically when selecting "Create Mesh".

Next on the window Hexpress, imported against the model that has been used as a parasolid format to speed up the mesh process that we do. After import is done, then adjust the position of the model that has been created using "Transform". This command serves to rotate or shift the location of the model we have created. Do not forget to remember the origin point of the object (point $0,0,0$ ). If you do not need to improve the position of the model then the next is done "Create Domain".

Domain is a solid part that is in a model. Computational domain or domain itself consists of topology and model geometry that we simulate. If described, the topology is a framework of a model, in general the topology states the definition of the volume or provides information of the interpersonal relationship on a model. While geometry is the real geometry of a model. Each surface of the model is described in triangulation and each curve is explained in detail through its element points.

Creating a domain on Hexpress (Numeca Fine Open) is very easy. Only need to enter the value of each parameter required. After entering the desired value then "apply" to see the domains created in the model.

After creating a domain on the model, next to do is meshing on the model. The first step before starting the mesh process is to define the boundary of the model. The boundary used in this study is solid (SOL) for dimple and surface plate, inlet (INT) and outlet (OUT) on the front and rear showing the incoming and outgoing sides of the domain, and external (EXT) to show the external side Which is modeled as the side in the water.

After making the selection of boundary condition, hereinafter is Initial Mesh. This mesh is useful This process makes the cell general and inaccurate of a model. This process only maps the general shape of the model.

Next we will pass Adapt to Geometry mesh. This process is an important process in meshing using hexpress. In this process the cells formed in the initial mesh stage will be adapted by the model. In other words, the formed cell is already resembling the model created. In this process there are 2 important things namely refinement and trimming. Refinement is to make cells that make the shape in the curve more specific. In the refinement process there are 3 things that can be done that is on the curve, surface and volume criterion. While the trimming process to eliminate all cells that exist outside the domain. Cells that are outside the domain will be automatically deleted and made adjustments with other cells.

After all stages are passed correctly (marked with green in mesh wizard box) mesh ready to be analyzed in Fine Open solver. After that the mesh results can be saved in .igg format indicating that the meshing process has been completed.

\section{Simulation}

In this simulation phase will be calculated as much as 9 times (9 speed or 9 numbers reynolds) that have been selected earlier in the design stage determination parameters.

This speed difference or reynold number represents a laminar, transitional, and turbulent flow state. So that later we can see the difference between these three states how the flow conditions and the magnitude of the drag that occurred.

This simulation depends on the number of cells created when performing mesh on the model. The more cells, the simulation or solver process will run longer to get the appropriate value or close to the desired criteria (reaching convergence).

This simulation process uses the solver of numeca fine open, so it needs to do some arrangement like in boundary condition, flow model, fluid model etc. The first parameter we will enter is general properties. General properties are the basic parameters for the simulation model which will involve optional models that can be added if needed.

Data collection is the reading stage of the simulation result through CFView contained in Numeca Fine Open software which is useful to read the simulation result. In this stage we collect $\mathrm{Cf}$ data or the coefficient of friction generated from the simulation of each model and its various speeds. In the data collection there will be 45 data generated by the simulation process. 


\section{RESULTS AND DISCUSSION}

All models that have been simulated, it can be done data retrieval needed to perform data analysis on this final task, So that obtained performance data from flat plate model

in velocity so as to obtain the average value to be compared in the graph and can be seen the changes in each type of flow (laminar, transition, and turbulent). that tested. The data is taken on the surface of the plate or surface with dimple or without dimple, the data to be taken is Cf.

The reading of the data referred to in this section is to read each segment owned by each model and its variation

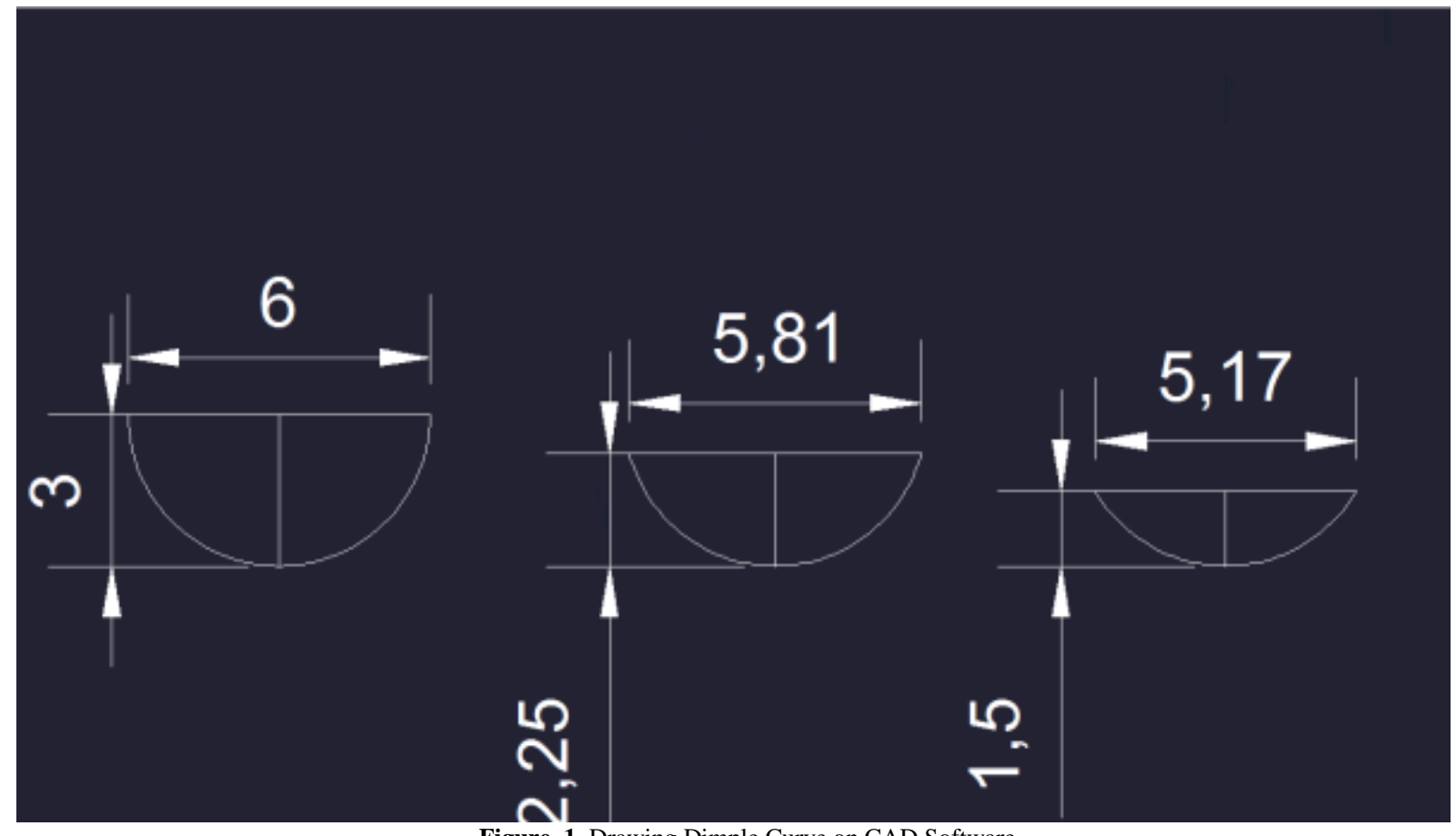

Figure. 1. Drawing Dimple Curve on CAD Software

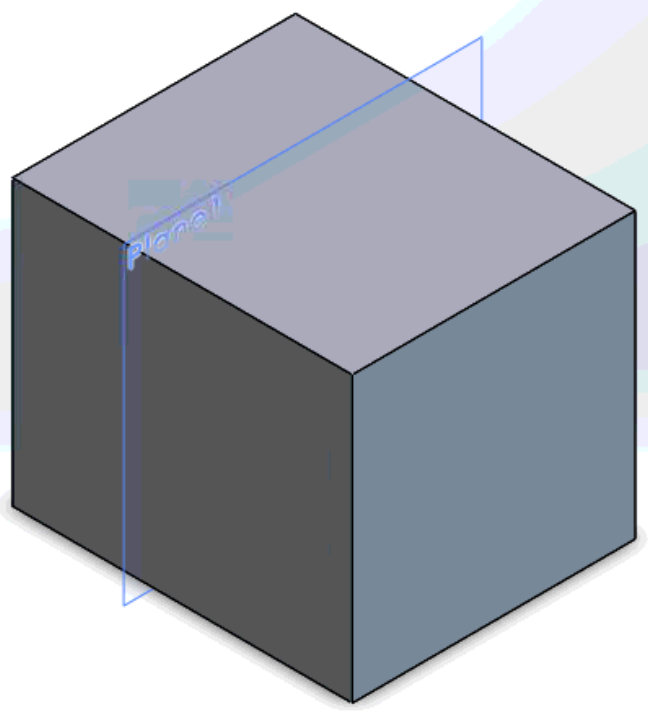

Figure. 2. Drawing Domain Block on CAD 3D. 


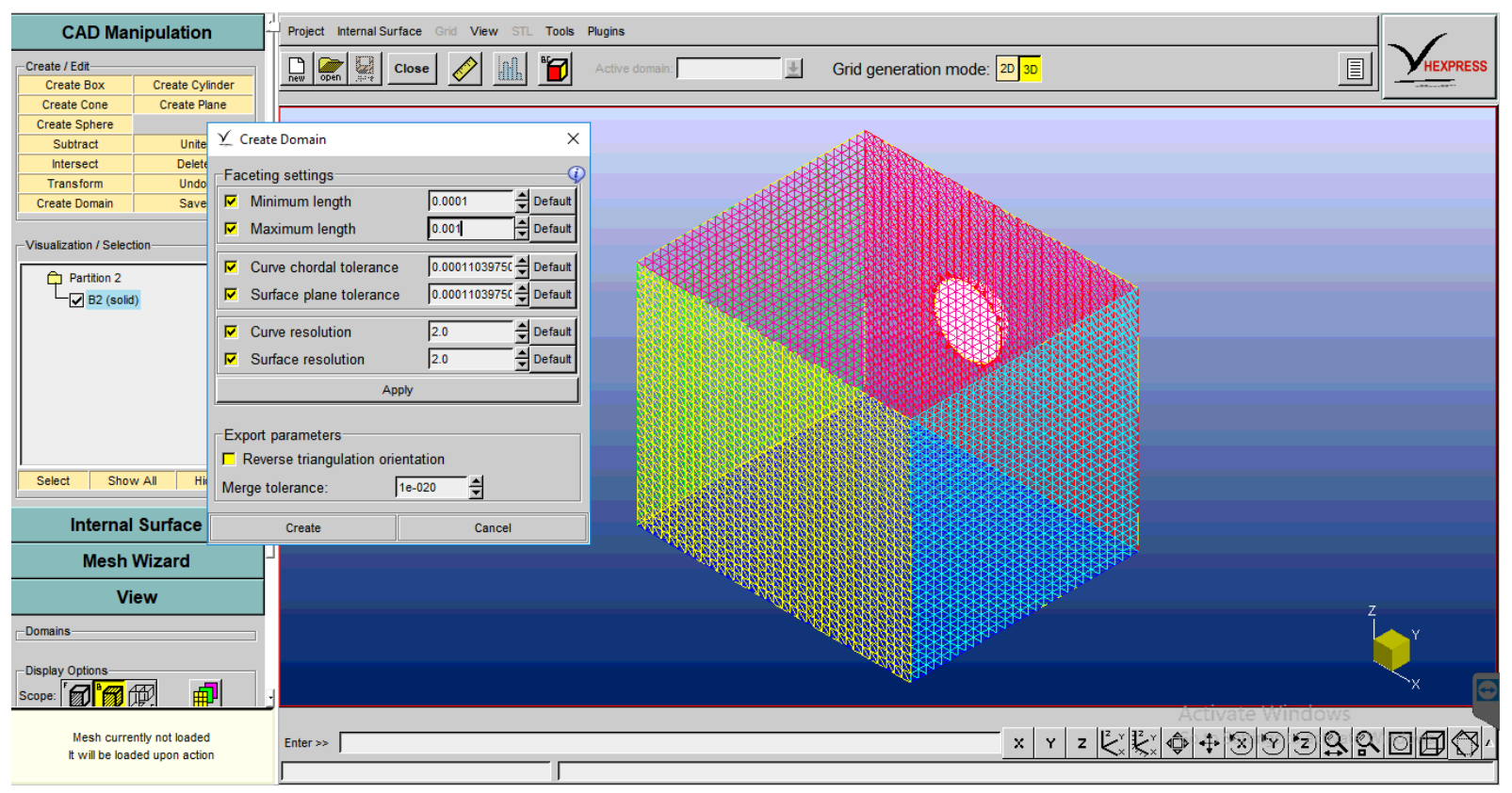

Figure. 3. Domain Creating on Numeca Fine Open "Hexpress"

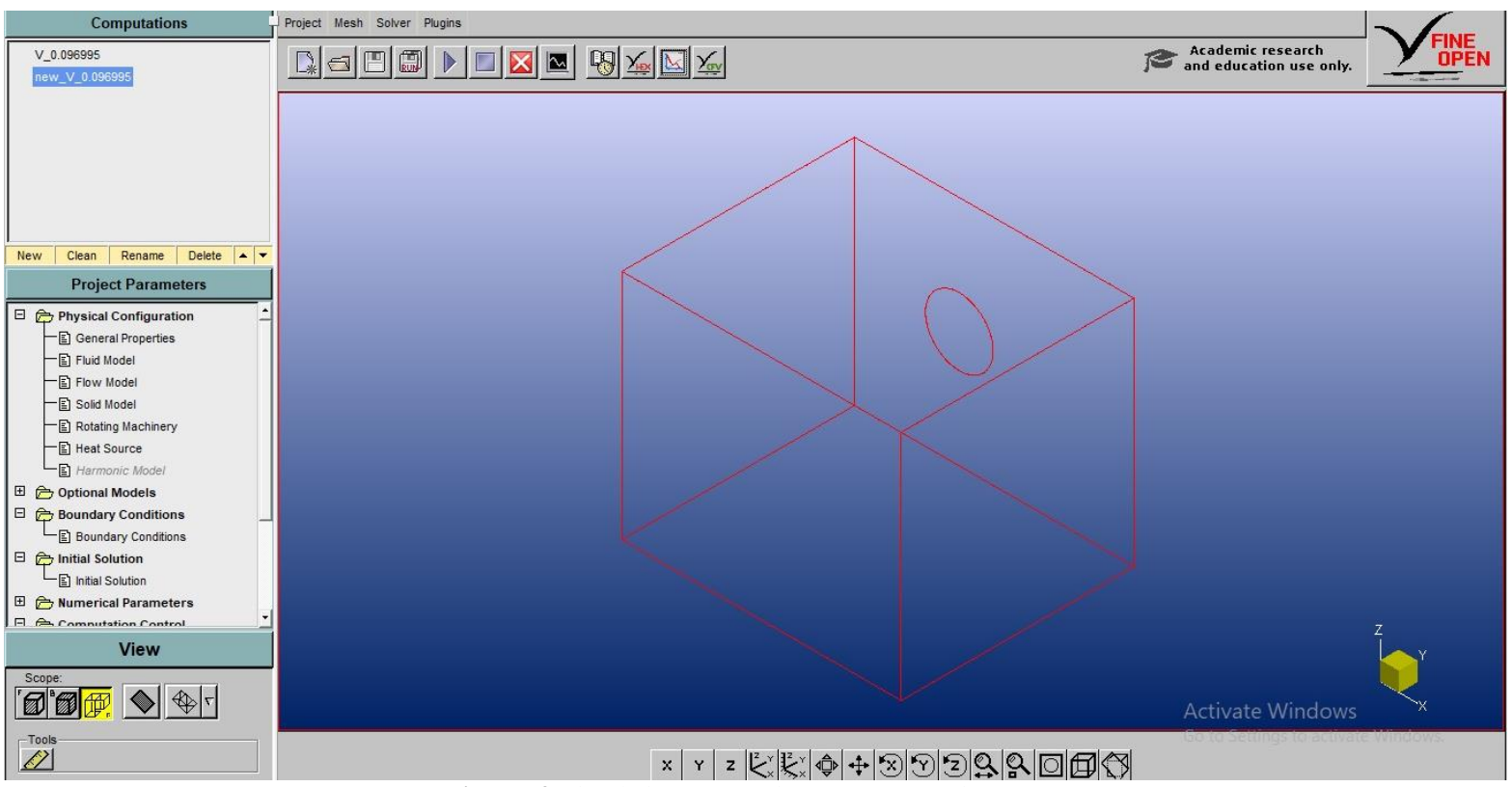

Figure. 4. Simulation Preparation on Numeca Fine Open.

From the above graph we separate into 3 segments where the first segment is on the left side of the hollow (dimple), the left side end can be called point A1. Then at the end of the first segment can be called point B1. From point $\mathrm{B} 1$ to the end of the basin is the second segment. The that it can be said the resistance is reduced. Then when passing the crosses (dimple) the value of Cf down to close to 0.03 after that the value of $\mathrm{Cf}$ rises back up to point D1 that is 0.05254 .

On the contours on the next side of the graph is a red color that represents the height of $\mathrm{Cf}$ at the inlet. Then it can be seen that Cf gradually decreases as it approaches the hole dimple. Then around the holes dimple can be seen end of the second segment is the point $\mathrm{C} 1$, and the last segment from point $\mathrm{C} 1$ to the end of the plate is point $\mathrm{D} 1$. The values at that point are $\mathrm{A} 1=0.18351, \mathrm{~B} 1=0.06896$, $\mathrm{C} 1=0.04114$, and $\mathrm{D} 1=0.05254$. Of the four values we can know that from point A1 to B1 Cf drastic decrease so that the surface color in the field is light blue. This indicates a decrease in $\mathrm{Cf}$ around the dimple opening followed by a split stream. However, the light blue color indicates that the boundary layer of the water stream is not released despite having passed the dimple. This indicates that the flow on the surface of the dimple is inseparable from the initial surface so it will not cause the vortex after passing the object (flat plate) is. 


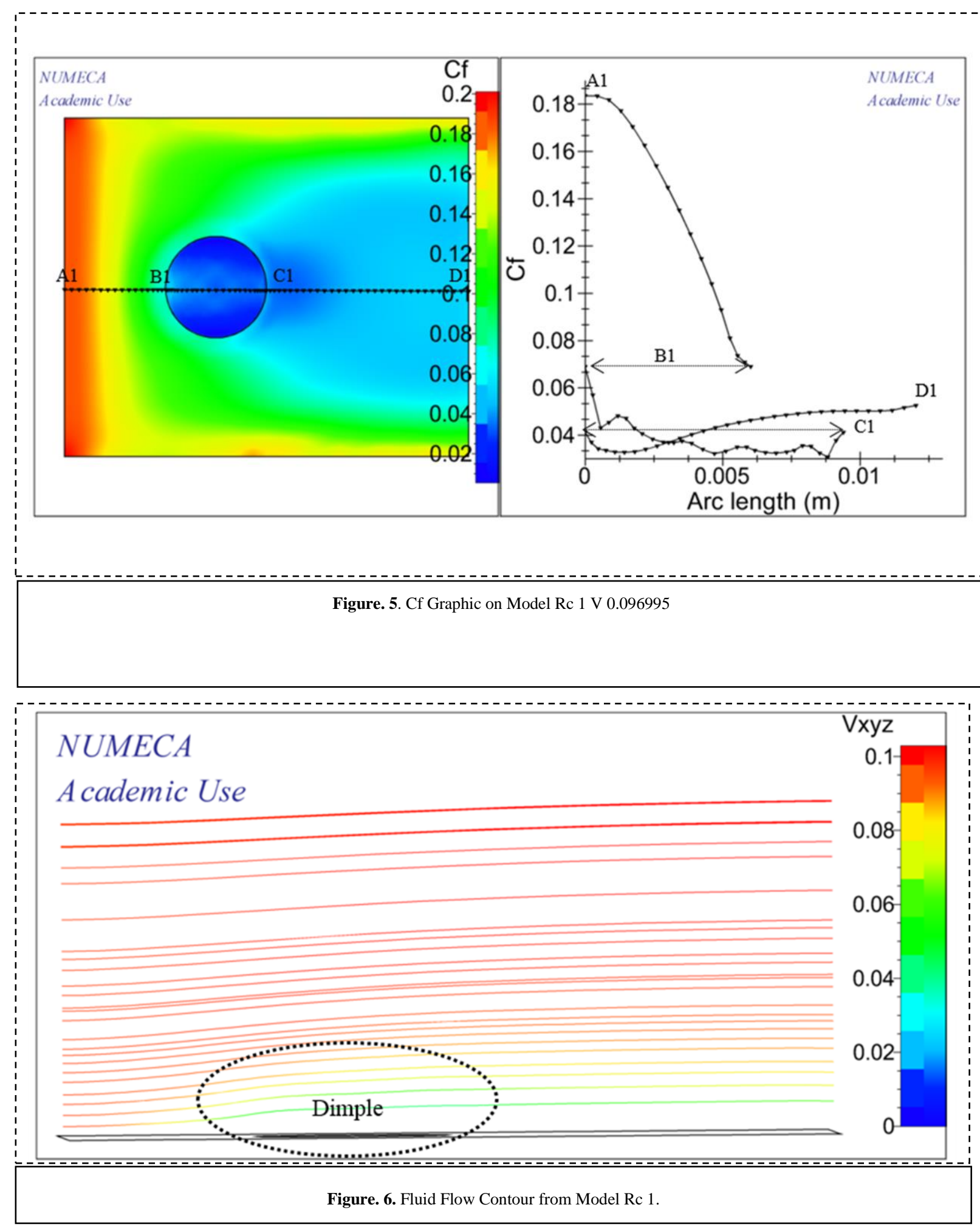

From above contour, fluid flow in beginning is uniform. Between above and below is smooth flow until dimple area which the stream moving above because dimple effect. In another model of flat plate with dimple, stream Why contour is not entering dimple hole in this model. It can be mesh of the model is not smooth so the stream looked entering dimple and effect on above surface of plate is making stream move upward. Velocity in surface around dimple area is gradually decrease and lowest velocity is detected on center of dimple that closing 0.02 . can looked entering dimple. 


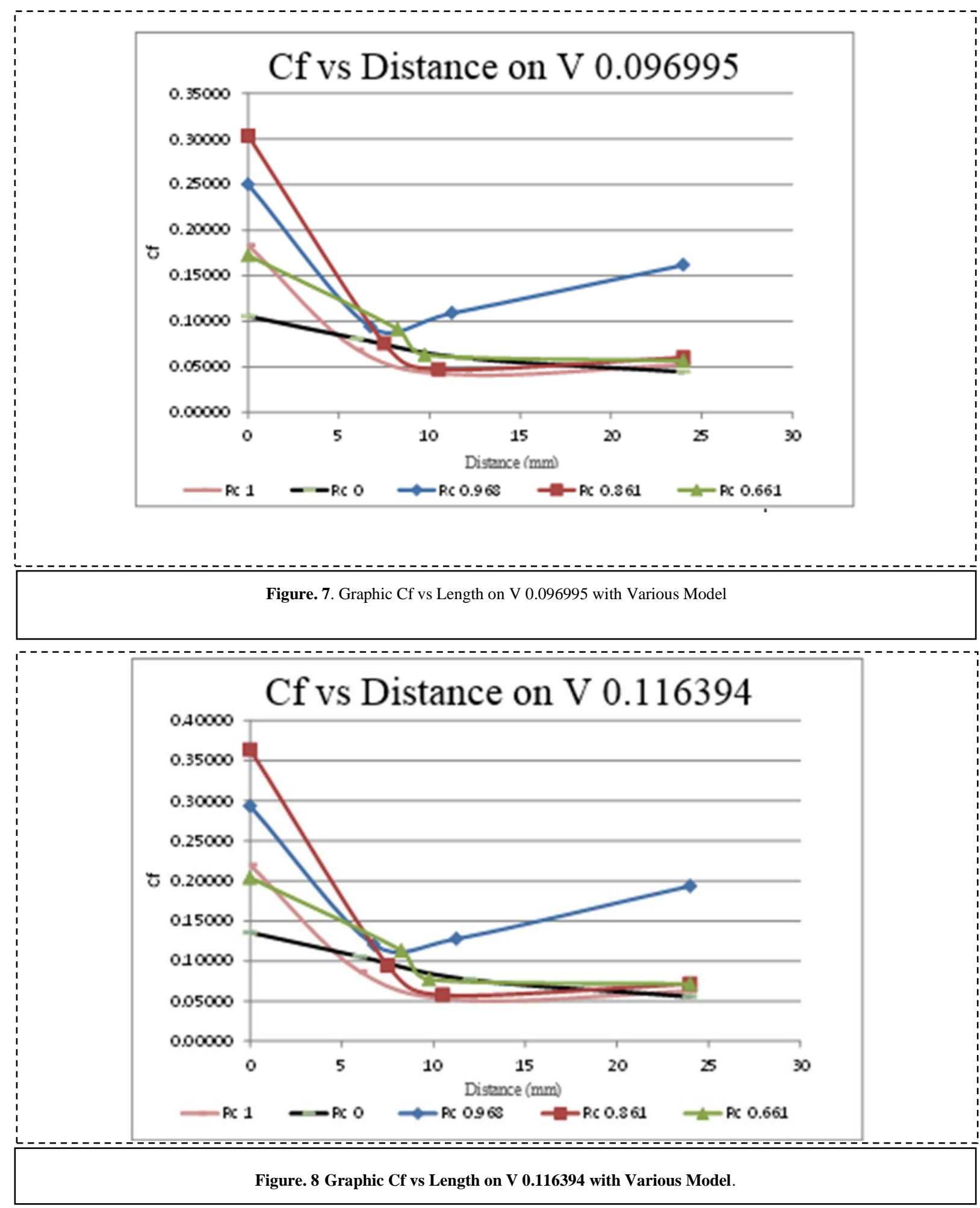

Above is a comparison graph of $\mathrm{Cf}$ with a flat plate length. Each curve is a representation of a simulated model. In the above graph taken at a speed of $0.096995 \mathrm{~m}$ / $\mathrm{s}$ or laminar lies in the flow. As explained in the previous chart which discusses the $\mathrm{Cf}$ of each simulation speed in each model. The average $\mathrm{Cf}$ value decreases as it moves away from the inlet. However, in model Rc 0.968 the value of $\mathrm{Cf}$ after dimple increases. This can be caused by a less precise meshing or simulated state that is not controlled so that the value of $\mathrm{Cf}$ is increasing. Then the Cf generated by the whole model looks uniform curve.
From this it can be seen that the simulation has been done properly and run the same at each variation of speed.

As with previous models, the reynold numbers that influence the determination of flow velocity make the difference between the curves above. The laminar stream is at the bottom of the graph ( 3 bottom curves) and then the transition flow continues at 3 graphs in the middle and last turbulent flow on the top 3 charts. 
Above is a comparison graph of $\mathrm{Cf}$ with a flat plate length. Each curve is a representation of a simulated model. The above graph is taken at a speed of $0.116394 \mathrm{~m}$ / $\mathrm{s}$ or lies in the transition stream. As explained in the previous chart which discusses the $\mathrm{Cf}$ of each simulation speed in each model. The average $\mathrm{Cf}$ value decreases as it moves away from the inlet. However, in model Rc 0.968 the value of Cf after dimple increases. This can be caused by a less precise meshing or simulated state that is not controlled so that the value of $\mathrm{Cf}$ is increasing. Then the Cf generated by the whole model looks uniform curve. From this it can be seen that the simulation has been done properly and run the same at each variation of speed.

If we observe further on the model curve Rc $1, \mathrm{Rc} 0.861$ becomes longer when under the Rc 0 model curve which is a flat plate without dimple. From this we can conclude that with different flow can change the value of $\mathrm{Cf}$ on plate with dimple to approach flat plate without dimple.

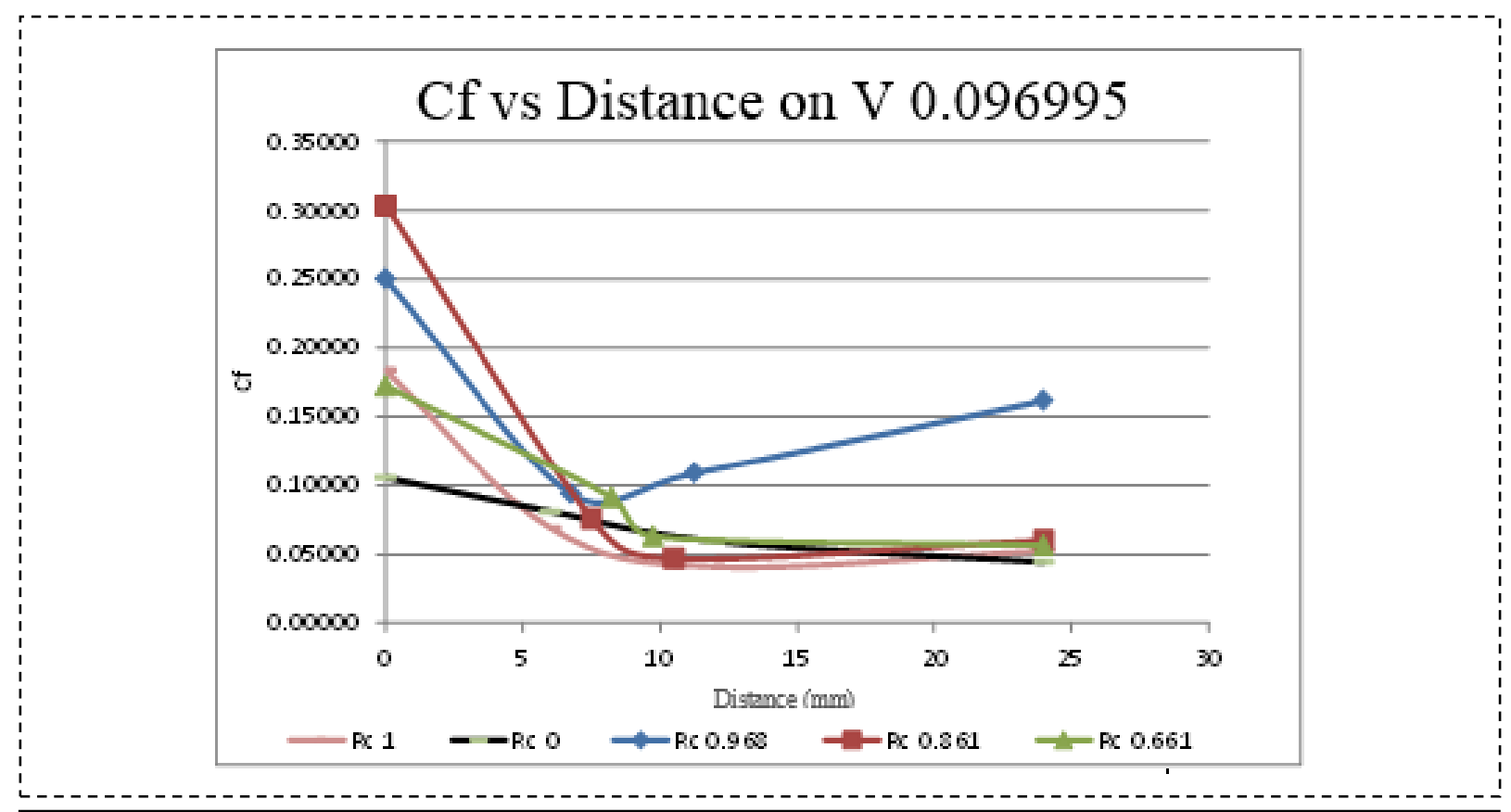

Figure. 9. Graphic Cf vs Length on V 0.198839 with Various Model.

Above is a comparison graph of $\mathrm{Cf}$ with a flat plate length. Each curve is a representation of a simulated model. The above graph is taken at a speed of $0.198839 \mathrm{~m}$ / $\mathrm{s}$ or lies in turbulent flow. As explained in the previous chart which discusses the $\mathrm{Cf}$ of each simulation speed in each model. The average $\mathrm{Cf}$ value decreases as it moves away from the inlet. However, in model Rc 0.968 the value of $\mathrm{Cf}$ after dimple increases. This can be caused by a less precise meshing or simulated state that is not controlled so that the value of $\mathrm{Cf}$ is increasing. Then the Cf generated by the whole model looks uniform curve. From this it can be seen that the simulation has been done properly and run the same at each variation of speed.

Similar to the comparison of the transition flow velocity, on the graph above the curve of model Rc 1, Rc 0.861 becomes longer when under model curve Rc 0 which is a flat plate without dimple. This indicates that the fluid flow type has an effect on the decrease in Cf.

\section{CONCLUSION}

From results of simulations and data analysis that written before, these are the conclusions:

1. Decrease coefficient of friction $(\mathrm{Cf})$ on plate with dimple. This decrease occurs only in the area around the dimple. This indicates that dimple affects the reduction of friction against flat plate by fluid flow.
2. Greatest decrease of coefficient of friction (Cf) is in model Rc 1which has the greatest ratio of concave among other models. The decrease was 0.083 (43\%) compared to flat plates without dimple.

3. With the ratio of concavity (Rc) 1 and flow between the turbulent and transitions state, make dimple be optimal in reducing the coefficient of friction (Cf). (Between 20-30\%).

4. Increase of speed making $\mathrm{Cf}$ of each models increase. But the difference between Cf plate with dimple and without dimple relatively decreased (between $20-30 \%$ ).

\section{REFERENCES}

[1] Harvald, S A, Resistance and Propultion of ships. Jhon Wiley and sons, toronto, Canada, 1983.

[2] Puji Kurniawan Nizar, Analisa Pengaruh Cekungan yang Diterapkan pada Pelat Datar Terhadap Aliran Fluida untuk Mendukung Teknologi Maritim Pendekatan CFD, Thesis. ME ITS Surabaya, 2011.

[3] Aziz Almujahidin, Mohammad, Eksperimen Pengaruh Cekungan yang Diterapkan pada Pelat Datar Terhadap Aliran Fluida, Skripsi. ME - ITS Surabaya, 2012.

[4] Anggraini, Meri. Viscous, Laminer, Turbulen. (downloaded in 6 Januari 2017) http://viscous-laminer-dan-turbulen.html

[5] Rosas, Hendri, 2009. Simulasi CFD pada Helm. (downloaded in 6 Februari 2017) www.cctonline.com/mekanika/tiki-index.php

[6] Rosas, Hendri, 2009. Aerodinamika pada Bola Golf (downloaded in 6 Februari 2017) www.cctonline.com/mekanika/tiki-index.php. 